\title{
New Estimates of Solution to Coupled System of Damped Wave Equations with Logarithmic External Forces
}

\author{
Loay Alkhalifa $\mathbb{D}^{1}$ and Khaled Zennir $\mathbb{D}^{1,2}$ \\ ${ }^{1}$ Department of Mathematics, College of Sciences and Arts, Ar-Rass, Qassim University, Saudi Arabia \\ ${ }^{2}$ Laboratoire de Mathématiques Appliquées et de Modélisation, Université 8 Mai 1945 Guelma, B.P. 401 Guelma 24000, Algeria \\ Correspondence should be addressed to Khaled Zennir; k.zennir@qu.edu.sa
}

Received 18 March 2021; Revised 30 March 2021; Accepted 2 April 2021; Published 10 April 2021

Academic Editor: Liliana Guran

Copyright (c) 2021 Loay Alkhalifa and Khaled Zennir. This is an open access article distributed under the Creative Commons Attribution License, which permits unrestricted use, distribution, and reproduction in any medium, provided the original work is properly cited.

In the paper, we consider new stability results of solution to class of coupled damped wave equations with logarithmic sources in $\mathbb{R}^{n}$. We prove a new scenario of stability estimates by introducing a suitable Lyapunov functional combined with some estimates.

\section{Introduction}

In the present paper, we consider an initial boundary value problem with damping terms and logarithmic sources, for $x$ $\in \mathbb{R}^{n}, t>0$

$$
\left\{\begin{array}{l}
\partial_{t t} v_{1}+b v_{2}=\phi(x) \Delta\left(v_{1}-\int_{0}^{t} \omega_{1}(t-p) v_{1}(p) d p\right)+k v_{1} \ln \left|v_{1}\right| \\
\partial_{t t} v_{2}+b v_{1}=\phi(x) \Delta\left(v_{2}-\int_{0}^{t} \omega_{2}(t-p) v_{2}(p) d p\right)+k v_{2} \ln \left|v_{2}\right|, \\
v_{1}(x, 0)=v_{10}(x), v_{2}(x, 0)=v_{20}(x), \\
\partial_{t} v_{1}(x, 0)=v_{11}(x), \partial_{t} v_{2}(x, 0)=v_{21}(x),
\end{array}\right.
$$

where $b>0, n \geq 3$, and $k$ is a small positive real number. The density function $\rho(x)>0$, for all $x \in \mathbb{R}^{n}$, where $(\phi(x))^{-1}=1 / \phi$ $(x) \equiv \rho(x)$, under homogeneous Drichlet boundary conditions.

A related initial boundary value problem was considered by Han in [1]:

$$
\begin{cases}u_{t t}+u_{t}-\Delta u+u+|u|^{2} u=u \ln |u|^{2}, & x \in \Omega, t \in[0, T), \\ u(x, 0)=u_{0}(x) u_{t}(x, 0)=u_{1}(x), & x \in \Omega, \\ u(x, t)=0, & x \in \partial \Omega, t \in[0, T),\end{cases}
$$

and the global existence of weak solutions was proved, for all $\left(u_{0}, u_{1}\right) \in H_{0}^{1} \times L^{2}$ in $\mathbb{R}^{3}$. The weak and strong damping terms in logarithmic wave equation

$$
\begin{cases}u_{t t}+\mu u_{t}-\Delta u-\omega \Delta u_{t}=u \ln |u|, & x \in \Omega, t \in(0, \infty), \\ u(x, 0)=u_{0}(x) u_{t}(x, 0)=u_{1}(x), & x \in \Omega, \\ u(x, t)=0, & x \in \partial \Omega, t \in(0, \infty)\end{cases}
$$

were introduced by Lian and $\mathrm{Xu}$ [2]. The global existence, asymptotic behavior, and blowup at three different initial energy levels (subcritical energy $E(0)<d$, critical initial energy $E(0)=d$, and the arbitrary high initial energy $E(0)>0(\omega=0)$ ) were proved. In [3], Al-Gharabli established explicit and general energy decay results for the problem

$$
\begin{cases}u_{t t}+\Delta^{2} u+u-\int_{0}^{t} g(t-s) \Delta^{2} u d s=k u \ln |u|, & x \in \Omega, t \in(0, \infty), \\ u(x, 0)=u_{0}(x) u_{t}(x, 0)=u_{1}(x), & x \in \Omega, \\ u(x, t)=\frac{\partial u}{\partial v}=0, & x \in \partial \Omega, t \in(0, \infty) .\end{cases}
$$


When the density $\phi(x) \neq 1$, Papadopoulos and Stavrakakis [4] considered the following semilinear hyperbolic initial value problem:

$$
u_{t t}+\phi(x) \Delta u+\delta u_{t}+\lambda f(u)=\eta(x),(x, t) \in \mathbb{R}^{n} \times \mathbb{R}^{+}
$$

The authors proved local existence of solutions and established the existence of a global attractor in the energy space $\mathscr{D}^{1,2}\left(\mathbb{R}^{n}\right) \times L_{g}^{2}\left(\mathbb{R}^{n}\right)$, where $(\phi(x))^{-1}:=g(x)$. Miyasita and Zennir [5] proved the global existence of the following viscoelastic wave equation:

$$
\begin{cases}u_{t t}+a u_{t}-\phi(x)\left(\Delta u+\omega \Delta u_{t}-\int_{0}^{t} g(t-s) \Delta u(s) d s\right)=u|u|^{p-1}, & x \in \mathbb{R}^{n}, t>0, \\ u(x, 0)=u_{0}(x), & x \in \mathbb{R}^{n}, \\ u_{t}(x, 0)=u_{1}(x), & x \in \mathbb{R}^{n} .\end{cases}
$$

The novelty of our work lies primarily in the use of a new condition between the weights of damping the external forces, where we outline the effects of the damping term with less conditions on the viscoelastic terms. We also propose logarithmic nonlinearities in sources and used classical arguments to estimate them. These nonlinearities make the problem very interesting in the application point of view. In order to compensate for the lack of classical Poincaré's inequality in $\mathbb{R}^{n}$, we use the weighted function to use generalized Poincaré's one. The main contribution of this paper is introduced in Theorem 8, where we obtain decay estimates with positive initial energy under a general assumption on the kernel. The rest of the paper is outline as follows. In Section 2, we give some preliminaries and our main results. In Section 3 , we will prove the general decay of energy to the problem.

\section{Preliminaries and Main Results}

We state some assumptions and definitions that will be useful in this paper. With respect to the relaxation functions $\omega_{1}, \omega_{2}$, we assume for $i=1,2$.

(H1) $\omega_{1}, \omega_{2} \in C^{1}\left(\mathbb{R}^{+}, \mathbb{R}^{+}\right)$satisfy for any $t \geq 0$,

$$
\omega_{i}(0)>0, \int_{0}^{\infty} \omega_{i}(p) d p=l_{i 0}<\infty, 1-\int_{0}^{t} \omega_{i}(p) d p=l_{i}>0
$$

(H2) There exist nonincreasing differentiable functions $\zeta_{1}, \zeta_{2}: \mathbb{R}^{+} \longrightarrow \mathbb{R}^{+}$that satisfy

$$
\zeta_{i}(t)>0, \omega_{i^{\prime}}(t) \leq-\zeta_{i}(t) \omega_{i}(t) \text { for } t \geq 0
$$

(H3) The function $\rho: \mathbb{R}^{n} \longrightarrow \mathbb{R}_{+}^{*}, \rho(x) \in C^{0, \gamma}\left(\mathbb{R}^{n}\right)$ with $\gamma \in(0,1)$ and $\rho \in L^{s}\left(\mathbb{R}^{n}\right) \cap L^{\infty}\left(\mathbb{R}^{n}\right)$, where $s=2 n / 2 n-q n+$ $2 q$
Definition 1 (see [4]). We define the function spaces of our problem and their norms as follows:

$$
\mathscr{H}=\left\{v \in L^{2 n /(n-2)}\left(\mathbb{R}^{n}\right) \mid \nabla v \in\left(L^{2}\left(\mathbb{R}^{n}\right)\right)^{n}\right\} .
$$

Let the function spaces $\mathscr{H}$ as the closure of $C_{0}^{\infty}\left(\mathbb{R}^{n}\right)$ with respect to the norm $\|v\|_{\mathscr{H}}=(v, v)_{\mathscr{H}}^{1 / 2}$ for the inner product:

$$
(v, w)_{\mathscr{H}}=\int_{\mathbb{R}^{n}} \nabla v \cdot \nabla w d x,
$$

and $L_{\rho}^{2}\left(\mathbb{R}^{n}\right)$ be defined with the norm $\|v\|_{L_{\rho}^{2}}=(v, v)_{L_{\rho}^{2}}^{1 / 2}$ for

$$
(v, w)_{L_{\rho}^{2}}=\int_{\mathbb{R}^{n}} \rho v w d x
$$

For general $q \in[1,+\infty), L_{\rho}^{q}\left(\mathbb{R}^{n}\right)$ is the weighted $L^{q}$ space under a weighted norm

$$
\|v\|_{L_{\rho}^{q}}=\left(\int_{\mathbb{R}^{n}} \rho|v|^{q} d x\right)^{1 / q} .
$$

To distinguish the usual $L^{q}$ space from the weighted one, we denote the standard $L^{q}$ norm by

$$
\|v\|_{q}=\left(\int_{\mathbb{R}^{n}}|v|^{q} d x\right)^{1 / q}
$$

$$
\begin{gathered}
\text { We denote an eigenpair }\left\{\left(\lambda_{j}, w_{j}\right)\right\}_{j \in \mathbb{N}} \subset \mathbb{R} \times \mathscr{H} \text { by } \\
-\phi(x) \Delta w_{j}=\lambda_{j} w_{j} \mathrm{x} \in \mathbb{R}^{\mathrm{n}}
\end{gathered}
$$

for any $j \in \mathbb{N}$. Then, according to [4],

$$
0<\lambda_{1} \leq \lambda_{2} \leq \cdots \leq \lambda_{j} \leq \cdots \uparrow+\infty
$$

holds and $\left\{w_{j}\right\}$ is a complete orthonormal system in $\mathscr{H}$.

Now, we introduce Sobolev embedding and generalized Poincaré's inequalities.

Lemma 2. Let $\rho$ satisfy (H3). Then, there are positive constants $C_{S}>0$ and $C_{P}>0$ that depend only on $n$ and $\rho$ such 
that

$$
\begin{gathered}
\|v\|_{2 n /(n-2)} \leq C_{S}\|v\|_{\mathscr{H}}, \\
\|v\|_{L_{\rho}^{2}} \leq C_{P}\|v\|_{\mathscr{H}}
\end{gathered}
$$

for $v \in \mathscr{H}$.

Lemma 3 (see Lemma 2.2 in [6]). Let $\rho$ satisfy (H3). Then, we have

$$
\begin{gathered}
\|v\|_{L_{\rho}^{q}} \leq C_{q}\|v\|_{\mathscr{H}}, \\
C_{q}=C_{S}\|\rho\|_{s}^{1 / q}
\end{gathered}
$$

for $v \in \mathscr{H}$, where $s=2 n /(2 n-q n+2 q)$ for $1 \leq q \leq 2 n /(n-2)$. by

The energy functional associated to problem (1) is given

$$
\begin{aligned}
\mathscr{E}(t)= & \frac{1}{2} \sum_{i=1}^{2}\left\|\partial_{t} v_{i}(t)\right\|_{L_{\rho}^{2}}^{2}+\frac{1}{2} \sum_{i=1}^{2}\left(1-\int_{0}^{t} \varrho_{i}(p) d p\right)\left\|\nabla v_{i}(t)\right\|^{2} \\
& +b\left\|v_{1}(t) v_{2}(t)\right\|^{2}+\frac{1}{2} \sum_{i=1}^{2}\left(\varrho_{i} \circ \nabla v_{i}\right)(t) \\
& -\frac{k}{2} \sum_{i=1}^{2} \int_{\mathbb{R}^{n}} \rho(x) v_{i}^{2} \ln \left|v_{i}\right| d x+\frac{k}{4} \sum_{i=1}^{2}\left\|v_{i}\right\|_{L_{\rho}^{2}}^{2} \\
\geq & \frac{1}{2} \sum_{i=1}^{2}\left\|\partial_{t} v_{i}(t)\right\|_{L_{\rho}^{2}}^{2}+\frac{1}{2} \sum_{i=1}^{2}\left(1-\int_{0}^{t}{\omega_{i}}_{i}(p) d p\right)\left\|\nabla v_{i}(t)\right\|^{2} \\
& +\frac{1}{2} \sum_{i=1}^{2}\left(\omega_{i} \circ \nabla v_{i}\right)(t)-\frac{k}{2} \sum_{i=1}^{2} \int_{\mathbb{R}^{n}} \rho(x) v_{i}^{2} \ln \left|v_{i}\right| d x \\
& +\frac{k}{4} \sum_{i=1}^{2}\left\|v_{i}\right\|_{L_{\rho}^{2},}^{2},
\end{aligned}
$$

where

$$
(\varpi \circ v)=\int_{0}^{t} \omega(t-p)\|v(t)-v(p)\|_{2}^{2} d p
$$

With direct differentiation of (18), using (1), we obtain

$$
\partial_{t} \mathscr{E}(t)=-\frac{1}{2} \sum_{i=1}^{2}\left(\omega_{i}(t)\left\|v_{i}\right\|_{\mathscr{H}}^{2}-\left(\omega_{i}^{\prime} \circ \nabla v_{i}\right)\right) \leq 0
$$

which let our system dissipative.

Lemma 4 (see [7]) (logarithmic Sobolev inequality). Lets $u$ be any function in $H_{0}^{1}(\Omega)$ and $a>0$ be any number. Then,

$\int_{\Omega} v^{2} \ln |v| d x \leq \frac{1}{2}\|v\|_{2}^{2} \ln \|v\|_{2}^{2}+\frac{a^{2}}{2 \pi}\|\nabla v\|_{2}^{2}-(1+\ln a)\|v\|_{2}^{2}$.
Lemma 5 (see [8]) (logarithmic Gronwall inequality). Let $c$ $>0, \gamma \in L^{1}\left(0, T ; \mathbb{R}^{+}\right)$, and assume that the function $\omega:[0, T$ ]$\longrightarrow[1, \infty)$ satisfies

$$
\omega(t) \leq c\left(1+\int_{0}^{t} \gamma(p) \omega(p) \ln \omega(p) d p\right), \quad 0 \leq t \leq T
$$

then

$$
\omega(t) \leq c \exp \left(c \int_{0}^{t} \gamma(p) d p\right), \quad 0 \leq t \leq T
$$

We define the following functionals

$$
\begin{aligned}
J(t)= & \frac{1}{2} \sum_{i=1}^{2}\left(1-\int_{0}^{t} \omega_{i}(p) d p\right)\left\|\nabla v_{i}(t)\right\|^{2} \\
& +\frac{1}{2} \sum_{i=1}^{2}\left(\omega_{i} \circ \nabla v_{i}\right)(t)-\frac{k}{2} \sum_{i=1}^{2} \int_{\mathbb{R}^{n}} \rho(x) v_{i}^{2} \ln \left|v_{i}\right| d x \\
& +\frac{k}{4} \sum_{i=1}^{2}\left\|v_{i}\right\|_{L_{\rho}^{2}}^{2}, \\
I(t)= & \frac{1}{2} \sum_{i=1}^{2}\left(1-\int_{0}^{t} \omega_{i}(p) d p\right)\left\|\nabla v_{i}(t)\right\|^{2} \\
& +\frac{1}{2} \sum_{i=1}^{2}\left(\omega_{i} \circ \nabla v_{i}\right)(t)-\frac{k}{2} \sum_{i=1}^{2} \int_{\mathbb{R}^{n}} \rho(x) v_{i}^{2} \ln \left|v_{i}\right| d x .
\end{aligned}
$$

Then, we introduce

$$
\begin{gathered}
W=\left\{\left(v_{1}, v_{2}\right): v_{1}, v_{2} \in \mathscr{H}: I(t)>0, J(t)<d\right\} \cup\{0\} . \\
\sum_{i=1}^{2}\left\|v_{i}\right\|^{2}<4 d \text { for all } t \in[0, T) .
\end{gathered}
$$

Lemma 6. Let $\left(v_{10}, v_{11}\right),\left(v_{20}, v_{21}\right) \in \mathscr{H} \times L_{\rho}^{2}\left(\mathbb{R}^{n}\right)$ such that 0 $<\mathscr{E}(0)<d$ and $I\left(t_{0}\right)>0$. Then, we have

$$
\left(v_{1}, v_{2}\right) \in W
$$

Theorem 7 (see [5]). Let $\left(v_{10}, v_{11}\right),\left(v_{20}, v_{21}\right) \in \mathscr{H} \times L_{\rho}^{2}\left(\mathbb{R}^{n}\right)$. Under the assumptions (H1)-(H3). Then, problem (1) has a global weak solution $u$ in the space

$$
\left(v_{1}, v_{2}\right) \in\left(C([0,+\infty) ; \mathscr{H}) \cap C^{1}\left([0,+\infty) ; L_{\rho}^{2}\left(\mathbb{R}^{n}\right)\right)\right)^{2} .
$$


Then, the main result in this paper is the general decay of energy to problem (1) which is given in the following theorem.

Theorem 8. Assume the assumptions $(\mathrm{H} 1)-(\mathrm{H} 3)$ hold and 0 $<\mathscr{E}(0)<$ d. Let $\left(v_{1}, v_{2}\right)$ be the weak solution of problem (1) with the initial data $\left(v_{10}, v_{11}\right),\left(v_{20}, v_{21}\right) \in \mathscr{H}\left(\mathbb{R}^{n}\right) \times L_{\rho}^{2}\left(\mathbb{R}^{n}\right)$. Then, there exist constant $\beta>0$ such that the energy $\mathscr{E}(t)$ defined by (18) satisfies for all $t>0$,

$$
\mathscr{E}(t) \leq \beta\left(1+\int_{t_{0}}^{t} \xi^{\varepsilon_{0}+1}(p) d p\right)^{-1 / \varepsilon_{0}}, \quad \varepsilon_{0} \in(0,1) .
$$

\section{Asymptotic Behavior for $\mathscr{E}(0)<d$}

The following technical lemmas are useful to prove the general decay of energy to problem (1).

Lemma 9. Under the assumptions in Theorem 8, then the functional $\Phi(t)$ defined by

$$
\Phi(t)=\int_{\mathbb{R}^{n}} \rho(x)\left(v_{1}(t) \partial_{t} v_{1}+v_{2}(t) \partial_{t} v_{2}(t)\right) d x d x
$$

satisfies for any $t \geq 0$,

$$
\begin{aligned}
\Phi^{\prime}(t) \leq & \sum_{i=1}^{2}\left\|\partial_{t} v_{i}(t)\right\|_{L_{\rho}^{2}}^{2}-\frac{1}{2} \sum_{i=1}^{2} l_{i}\left\|\nabla v_{i}(t)\right\|^{2} \\
& +\sum_{i=1}^{2} \frac{1-l_{i}}{4 \varepsilon}\left(\omega_{i} \circ \nabla v_{i}\right)(t) \\
& +k \sum_{i=1}^{2} \int_{\mathbb{R}^{n}} \rho(x) v_{i}^{2} \ln \left|v_{i}\right| d x
\end{aligned}
$$

Proof. We differentiate $\Phi(t)$, using (1), we can get

$$
\begin{aligned}
\Phi^{\prime}(t)= & \sum_{i=1}^{2}\left\|\partial_{t} v_{i}\right\|_{L_{\rho}^{2}}^{2}-\sum_{i=1}^{2}\left\|\nabla v_{i}\right\|^{2} \\
& +\sum_{i=1}^{2} \int_{\mathbb{R}^{n}} \nabla v_{i}(t) \cdot \int_{0}^{t} \Phi_{i}(t-p) \nabla v_{i}(p) d p d x \\
& -2 b \int_{\mathbb{R}^{n}} \rho(x) v_{1} v_{2} d x+k \sum_{i=1}^{2} \int_{\mathbb{R}^{n}} \rho(x) v_{i}^{2} \ln \left|v_{i}\right| d x
\end{aligned}
$$

It follows from Young and Poincaré's inequality that for any $\varepsilon>0$,

$$
\begin{aligned}
\int_{\mathbb{R}^{n}} & \nabla v_{i}(t) \cdot \int_{0}^{t} \omega_{i}(t-p) \nabla v_{i}(p) d p d x \\
= & \int_{\mathbb{R}^{n}} \nabla v_{i}(t) \cdot \int_{0}^{t} \omega_{i}(t-p)\left(\nabla v_{i}(p)-\nabla v_{i}(t)\right) d p d x \\
& +\int_{0}^{t} \omega_{i}(p) d p\left\|\nabla v_{i}(t)\right\|_{2}^{2} \\
\leq & \left(1-l_{i}\right)\left\|\nabla v_{i}\right\|^{2}+\varepsilon\left\|\nabla v_{i}\right\|_{2}^{2}+\frac{1}{4 \varepsilon} \int_{\mathbb{R}^{n}} \\
& \cdot\left(\int_{0}^{t} \omega_{i}(t-p)\left(\nabla v_{i}(p)-\nabla v(t)\right) d p\right)^{2} d x \\
\leq & \left(1-l_{i}+\varepsilon\right)\left\|\nabla v_{i}\right\|_{2}^{2}+\frac{1-l_{i}}{4 \varepsilon}\left(\omega_{i} \circ \nabla v_{i}\right)(t) .
\end{aligned}
$$

Exploit Young and Poincaré's inequalities to estimate

$$
2 b \int_{\mathbb{R}^{n}} \rho(x) v_{1} v_{2} d x \leq \varepsilon \mathcal{c}_{*}\left\|\nabla v_{i}\right\|_{L_{\rho}^{2}}^{2}+\frac{c_{*}}{4 \varepsilon}\left\|\nabla v_{2}\right\|_{L_{\rho}^{2}}^{2} .
$$

Inserting (32)-(33) into (31) yields for any $\varepsilon>0$,

$$
\begin{aligned}
\Phi^{\prime}(t) \leq & \sum_{i=1}^{2}\left\|\partial_{t} v_{i}(t)\right\|_{L_{\rho}^{2}}^{2}-\sum_{i=1}^{2}\left(l-\varepsilon-\varepsilon c_{*}\right)\left\|\nabla v_{i}(t)\right\|^{2} \\
& +\sum_{i=1}^{2} \frac{1-l_{i}}{4 \varepsilon}\left(\omega_{i} \circ \nabla v_{i}\right)(t) \\
& +k \sum_{i=1}^{2} \int_{\mathbb{R}^{n}} \rho(x) v_{i}^{2} \ln \left|v_{i}\right| d x
\end{aligned}
$$

Taking $\varepsilon>0$ small enough in (34) such that

$$
l_{i}-\varepsilon-\varepsilon c_{*}>\frac{l}{2}
$$

The proof is hence complete.

Lemma 10. Under the assumptions in Theorem 8, then the functional $\psi(t)$ defined by

$$
\begin{aligned}
\psi(t)= & -\int_{\mathbb{R}^{n}} \rho(x) \partial_{t} v_{1}(t) \int_{0}^{t} \omega_{1}(t-p)\left(v_{1}(t)-v_{1}(p)\right) d p d x \\
& -\int_{\mathbb{R}^{n}} \rho(x) \partial_{t} v_{2}(t) \int_{0}^{t} \omega_{2}(t-p)\left(v_{2}(t)-v_{2}(p)\right) d p d x
\end{aligned}
$$


satisfies for any $\delta>0$,

$$
\begin{aligned}
\psi^{\prime}(t) \leq & \sum_{i=1}^{2} \delta\left[\left(1-l_{i}\right)^{2}+1+b c_{*}\right]\left\|\nabla v_{i}(t)\right\|^{2} \\
& -\sum_{i=1}^{2}\left[\left(\int_{0}^{t} \varrho_{i}(s) d p\right)-2 \delta\right]\left\|\partial_{t} v_{i}(t)\right\|_{L_{\rho}^{2}}^{2} \\
& +C \sum_{i=1}^{2}\left(\int_{0}^{t} \varrho_{i}(s) d p\right)\left(\varrho_{i} \circ \nabla v_{i}\right)(t) \\
& -\frac{\varrho_{i}(0) c_{*}}{4 \delta} \sum_{i=1}^{2}\left(\varpi_{i}^{\prime} \circ \Delta v_{i}\right)(t) \\
& +c_{\varepsilon_{0}} \sum_{i=1}^{2}\left(\varpi_{i} \circ \nabla v_{i}\right)^{1 /\left(1+\varepsilon_{0}\right)} .
\end{aligned}
$$

Proof. Taking the derivative of $\psi(t)$ and using (1), we conclude that

$$
\begin{aligned}
\psi^{\prime}(t)= & \sum_{i=1}^{2} \int_{\mathbb{R}^{n}} \nabla v_{i}(t) \int_{0}^{t} \omega_{i}(t-p)\left(\nabla v_{i}(t)-\nabla v_{i}(p)\right) d p d x \\
& -\int_{\mathbb{R}^{n}}\left(\int_{0}^{t} \Phi_{1}(t-p) \nabla v_{1}(p) d p\right) \\
& \cdot\left(\int_{0}^{t} \omega_{1}(t-p)\left(\nabla v_{1}(t)-\nabla v_{1}(p)\right) d p\right) d x \\
& -\int_{\mathbb{R}^{n}}\left(\int_{0}^{t} \omega_{2}(t-p) \nabla v_{2}(p) d p\right) \\
& \cdot\left(\int_{0}^{t} \omega_{2}(t-p)\left(\nabla v_{2}(t)-\nabla v_{2}(p)\right) d p\right) d x \\
& +b \int_{\mathbb{R}^{n}} \rho(x) v_{2} \int_{0}^{t} \Phi_{1}(t-p)\left(v_{1}(t)-v_{1}(p)\right) d p d x \\
& +b \int_{\mathbb{R}^{n}} \rho(x) v_{1} \int_{0}^{t} \Phi_{2}(t-p)\left(v_{2}(t)-v_{2}(p)\right) d p d x \\
& -k \sum_{i=1}^{2} \int_{\mathbb{R}^{n}} \rho(x) v_{i} \ln \left|v_{i}\right| \int_{0}^{t} \omega_{i}(t-p)\left(v_{i}(t)-v_{i}(p)\right) d p d x \\
& -\sum_{i=1}^{2} \int_{0}^{t} \Phi_{i}(p) d p\left\|\partial_{t} v_{i}\right\|_{L_{\rho}^{2}}^{2} \\
& -\sum_{i=1}^{2} \int_{\mathbb{R}^{n}} \rho(x) \partial_{t} v_{i} \int_{0}^{t} \omega_{i}^{\prime}(t-p)\left(v_{i}(t)-v_{i}(p)\right) d p d x .
\end{aligned}
$$

We then use Young and Poincaré's inequalities; we can get for any $\delta>0$,

$$
\begin{aligned}
\int_{\mathbb{R}^{n}} \nabla v_{i}(t) \int_{0}^{t} \varrho_{i}(t-p)\left(\nabla v_{i}(t)-\nabla v_{i}(p)\right) d p d x \\
\leq \delta\left\|\nabla v_{i}\right\|^{2}+\frac{1}{4 \delta}\left(\int_{0}^{t} \varrho_{i}(p) d p\right)\left(\varrho_{i} \nabla \nabla v_{i}\right)(t) .
\end{aligned}
$$

The second and third terms can be treated as

$$
\begin{gathered}
\int_{\mathbb{R}^{n}}\left(\int_{0}^{t} \omega_{i}(t-p) \nabla v_{i}(p) d p\right)\left(\int_{0}^{t} \omega_{i}(t-p)\left(\nabla v_{i}(t)-\nabla v_{i}(p)\right) d p\right) d x \\
\leq \delta\left(1-l_{i}\right)^{2}\left\|\nabla v_{i}\right\|^{2}+\left(1+\frac{1}{4 \delta}\right)\left(\int_{0}^{t} \omega_{i}(p) d p\right)\left(\varrho_{i} \circ \nabla v_{i}\right)(t) .
\end{gathered}
$$

The fourth and fifth terms will be estimated by

$$
\begin{gathered}
\int_{\mathbb{R}^{n}} \rho(x) v_{2} \int_{0}^{t} \varpi_{1}(t-p)\left(v_{1}(t)-v_{1}(p)\right) d p d x \\
\leq \delta c_{*}\left\|\nabla v_{2}\right\|^{2}+\frac{c_{*}}{4 \delta}\left(\int_{0}^{t} \varrho_{1}(p) d p\right)\left(\varpi_{1} \circ \nabla v_{1}\right)(t), \\
\int_{\mathbb{R}^{n}} \rho(x) v_{1} \int_{0}^{t} \varpi_{2}(t-p)\left(v_{2}(t)-v_{2}(p)\right) d p d x \\
\leq \delta c_{*}\left\|\nabla v_{1}\right\|^{2}+\frac{c_{*}}{4 \delta}\left(\int_{0}^{t} \varpi_{2}(p) d p\right)\left(\omega_{2} \circ \nabla v_{2}\right)(t),
\end{gathered}
$$

respectively.

For the last term, we have

$$
\begin{aligned}
& \int_{\mathbb{R}^{n}} \rho(x) \partial_{t} v_{i} \int_{0}^{t} \omega_{i}^{\prime}(t-p)\left(v_{i}(t)-v_{i}(p)\right) d p d x \\
& \quad \leq \delta\left\|\partial_{t} v_{i}\right\|_{L_{\rho}^{2}}^{2}+\frac{c_{*}}{4 \delta}\left(\int_{0}^{t}-\omega_{i}^{\prime}(p) d p\right)\left(\omega_{i}^{\prime} \mathrm{o} \nabla v_{i}\right)(t) \\
& \quad \leq \delta\left\|\partial_{t} v_{i}\right\|_{L_{\rho}^{2}}^{2}-\frac{\omega_{i}(0) c_{*}}{4 \delta}\left(\omega_{i}^{\prime} \mathrm{\circ} \nabla v_{i}\right)(t) .
\end{aligned}
$$

Let $\varepsilon_{0} \in(0,1)$ and $g(s)=s^{\varepsilon_{0}}(|\ln s|-s)$. Notice that $g$ is continous on $(0, \infty)$, its limit at 0 is 0 , and its limit at $\infty$ is $-\infty$. Then, $g$ has a maximum $m_{\varepsilon_{0}}$ on $[0, \infty)$, so the following inequality holds

$$
s|\ln s| \leq s^{2}+m_{\varepsilon_{0}} s^{1-\varepsilon_{0}}, \quad \text { for all } s>0 .
$$

Using the Cauchy-Schwartz's inequality and applying (43), yields, for any $\delta>0$,

$$
\begin{aligned}
k \int_{\mathbb{R}^{n}} \rho(x) v_{i} \ln \left|v_{i}\right| \int_{0}^{t} \Phi_{i}(t-p)\left(v_{i}(t)-v_{i}(p)\right) d p d x \\
\leq k \int_{\mathbb{R}^{n}} \rho(x)\left(v_{i}^{2}+m_{\varepsilon_{0}}\left|v_{i}\right|^{1-\varepsilon_{0}}\right) \\
\quad \cdot\left|\int_{0}^{t} \varrho_{i}(t-p)\left(v_{i}(t)-v_{i}(p)\right) d p d x\right| \\
\leq c \int_{\mathbb{R}^{n}} \rho(x) v_{i}^{2}\left|\int_{0}^{t} \omega_{i}(t-p)\left(v_{i}(t)-v_{i}(p)\right) d p d x\right| \\
\quad+\delta\left\|v_{i}\right\|_{L_{\rho}^{2}}^{2}+\int_{\mathbb{R}^{n}}\left|\int_{0}^{t} \varrho_{i}(t-p)\left(v_{i}(t)-v_{i}(p)\right) d p d x\right|^{2 /\left(1+\varepsilon_{0}\right)} \\
\leq \delta c_{*}\left\|\nabla v_{i}\right\|_{2}^{2}+\frac{1}{4 \delta}\left(\varrho_{i} \circ \nabla v_{i}\right)(t)+c_{\varepsilon_{0}}\left(\varrho_{i} \circ \nabla v_{i}\right)^{1 /\left(1+\varepsilon_{0}\right)} .
\end{aligned}
$$


Combining (39)-(44) with (39) gives us (37) with

$$
C=\frac{b c_{*}+2}{4 \delta}+2 \delta
$$

Therefore, the proof is complete.

Now, we define a Lyapunov functional $\mathscr{L}(t)$ by

$$
\mathscr{L}(t)=M \mathscr{E}(t)+\varepsilon_{1} \Phi(t)+\varepsilon_{2} \psi(t)
$$

where $M, \varepsilon_{1}$, and $\varepsilon_{2}$ are positive constants which will be taken later.

It is easy to see that $\mathscr{L}(t)$ and $\mathscr{E}(t)$ are equivalent in the sense that there exist two positive constants $\beta_{1}$ and $\beta_{2}$ such that

$$
\beta_{1} \mathscr{E}(t) \leq \mathscr{L}(t) \leq \beta_{2} \mathscr{E}(t)
$$

Remark 11 (see [3]). Since $\zeta_{i}$ is nonincreasing, we have

$$
\left.\zeta_{i}(t)\left(\omega_{i} \circ \nabla v_{i}\right)\right)^{1 /\left(1+\varepsilon_{0}\right)} \leq C\left(-\mathscr{E}^{\prime}(t)\right)^{1 /\left(1+\varepsilon_{0}\right)} .
$$

Proof of Theorem 8. For any fixed $t_{0}>0$, we have for any $t$ $\geq t_{0}$,

$$
\int_{0}^{t} \omega_{i}(p) d p \geq \int_{0}^{t_{0}} \omega_{i}(p) d p:=\omega_{i 0} .
$$

It follows from (37), (30), and (20) that

$$
\begin{aligned}
\mathscr{L}^{\prime}(t)= & M \mathscr{E}^{\prime}(t)+\varepsilon_{1} \Phi^{\prime}(t)+\psi^{\prime}(t) \\
\leq & -\sum_{i=1}^{2}\left(\varrho_{i 0}-2 \delta-\varepsilon_{1}\right)\left\|\partial_{t} v_{i}(t)\right\|_{L_{\rho}^{2}}^{2} \\
& -\sum_{i=1}^{2}\left[\frac{l_{i}}{2} \varepsilon_{1}-\delta\left(\left(1-l_{i}\right)^{2}+1+b c_{*}\right)\right]\left\|\nabla v_{i}(t)\right\|_{2}^{2} \\
& +\sum_{i=1}^{2}\left[C_{1} \varepsilon_{1}+C l_{i}\right]\left(\omega_{i} \circ \nabla v_{i}\right)(t)-\frac{M}{2} \sum_{i=1}^{2}{\omega_{i}}_{i}(t)\left\|v_{i}(t)\right\|^{2} \\
& \left.+\varepsilon_{1} k \sum_{i=1}^{2} \int_{\mathbb{R}^{n}} \rho(x) v_{i}^{2} \ln \left|v_{i}\right| d x+\varepsilon_{1} c_{\varepsilon_{0}} \sum_{i=1}^{2}\left(\varrho_{i} \circ \nabla v_{i}\right)\right)^{1 /\left(1+\varepsilon_{0}\right)} \\
& +C_{3} \sum_{i=1}^{2}\left(\varpi_{i}^{\prime} \circ \nabla v_{i}\right)(t) .
\end{aligned}
$$

Using the logarithmic Sobolev inequality, we have

$$
\begin{aligned}
\mathscr{L}^{\prime}(t) \leq & -\sum_{i=1}^{2}\left(\omega_{i 0}-2 \delta-\varepsilon_{1}\right)\left\|\partial_{t} v_{t}(i)\right\|_{L_{\rho}^{2}}^{2} \\
& +C_{3} \sum_{i=1}^{2}\left(\omega_{i}^{\prime} \circ \nabla v_{i}\right)(t) \\
& -\sum_{i=1}^{2}\left[\frac{l_{i}}{2} \varepsilon_{1}-\delta\left(\left(1-l_{i}\right)^{2}+1+b c_{*}-\varepsilon_{1} k \frac{\alpha^{2}}{2 \pi}\right)\right] \\
& \left\|\nabla v_{i}(t)\right\|_{2}^{2}+\sum_{i=1}^{2}\left[C_{1} \varepsilon_{1}+C l_{i}\right]\left(\omega_{i} \circ \nabla v_{i}\right)(t) \\
& -\frac{M}{2} \sum_{i=1}^{2} \Phi_{i}(t)\left\|v_{i}(t)\right\|^{2}+\varepsilon_{1} k \frac{1}{2} \sum_{i=1}^{2}\left\|v_{i}\right\|_{2}^{2} \ln \left\|v_{i}\right\|_{2}^{2} \\
& \left.-\varepsilon_{1} k(1+\ln \alpha) \sum_{i=1}^{2}\left\|v_{i}\right\|_{2}^{2}+\varepsilon_{1} c_{\varepsilon_{0}} \sum_{i=1}^{2}\left(\omega_{i} \circ \nabla v_{i}\right)\right)^{1 /\left(1+\varepsilon_{0}\right)} .
\end{aligned}
$$

Recalling (18) and $\mathscr{E}(t) \leq \mathscr{E}(0)<d$, we get

$\ln \|v\|_{2}^{2}<\ln \left(\frac{4}{k} \mathscr{E}(t)\right)<\ln \left(\frac{4}{k} \mathscr{E}(0)\right)<\ln \left(\frac{4}{k} d\right)$.

Now, we take $\varepsilon_{1}>0$ small enough so that

$$
\left(\omega_{i 0}-2 \delta-\varepsilon_{1}\right)>0 \text {. }
$$

For any fixed $\varepsilon_{1}>0$, we pick $\delta>0$ so small that

$$
\frac{l_{i}}{2} \varepsilon_{1}-\delta\left(\left(1-l_{i}\right)^{2}+1\right)>\frac{l_{i}}{4} \varepsilon_{1}
$$

On the other hand, we choose $M>0$ large enough so that (47) holds, and further

$$
C_{3}=\frac{M}{2}-\frac{\omega_{i}(0)}{4 \delta}>0
$$

We can conclude that there exist two positive constant $m$ and $C^{\prime}$ such that

$$
\left.\mathscr{L}^{\prime}(t) \leq-m \mathscr{E}(t)+C^{\prime} \sum_{i=1}^{2}\left(\varrho_{i} \circ \nabla v_{i}\right)(t)+\varepsilon_{1} c_{\varepsilon_{0}} \sum_{i=1}^{2}\left(\varrho_{i} \circ \nabla v_{i}\right)\right)^{1 /\left(1+\varepsilon_{0}\right)} .
$$

Multiplying (56) by $\zeta(t)=\min \left\{\zeta_{1}, \zeta_{2}\right\}$ by (H2) and use the fact that

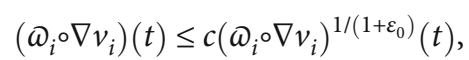

and (48), we get

$$
\zeta(t) \mathscr{L}^{\prime}(t) \leq-m \zeta(t) \mathscr{E}(t)+c\left(-\mathscr{E}^{\prime}(t)\right)^{1 /\left(1+\varepsilon_{0}\right)} .
$$


Multiply (58) by $\zeta^{\varepsilon_{0}}(t) \mathscr{E}^{\varepsilon_{0}}(t)$ and recall that $\zeta^{\prime}(t) \leq 0$ to obtain

$\zeta^{\varepsilon_{0}+1}(t) \mathscr{E}^{\varepsilon_{0}}(t) \mathscr{L}^{\prime}(t) \leq-m \zeta^{\varepsilon_{0}}(t) \mathscr{E}^{\varepsilon_{0}+1}(t)+c(\zeta \mathscr{E})^{\varepsilon_{0}}(t)\left(-\mathscr{E}^{\prime}(t)\right)^{1 /\left(1+\varepsilon_{0}\right)}$.

Using Young's inequality, for any $\delta>0$,

$$
\begin{aligned}
\zeta^{\varepsilon_{0}+1}(t) \mathscr{E}^{\varepsilon_{0}}(t) \mathscr{L}^{\prime}(t) \leq & -m \zeta^{\varepsilon_{0}+1}(t) \mathscr{E}^{\varepsilon_{0}+1}(t) \\
& +c\left(\delta \zeta^{\varepsilon_{0}+1}(t) \mathscr{E}^{\varepsilon_{0}+1}(t)-c_{\delta} \mathscr{E}^{\prime}(t)\right) \\
\leq & -(m-\delta c) \zeta^{\varepsilon_{0}+1}(t) \mathscr{E}^{\varepsilon_{0}+1}(t)-c \mathscr{E}^{\prime}(t),
\end{aligned}
$$

which implies

$$
\left(\zeta^{\varepsilon_{0}+1} \mathscr{C}^{\varepsilon_{0}} \mathscr{L}+c \mathscr{C}\right)(t) \leq-(m-\delta c) v^{\varepsilon_{0}+1}(t) \mathscr{E}^{\varepsilon_{0}+1}(t)
$$

It is clear that to get

$$
\mathscr{L}_{1}(t)=\left(\zeta^{\varepsilon_{0}+1} \mathscr{E}^{\varepsilon_{0}} \mathscr{L}+c \mathscr{E}\right) \sim \mathscr{E}(t)
$$

By using (61) and $\zeta^{\prime}(t) \leq 0$, we arrive at

$$
\mathscr{L}_{1^{\prime}}(t)=\left(\zeta^{\varepsilon_{0}+1} \mathscr{E}^{\varepsilon_{0}} \mathscr{L}+c \mathscr{E}\right) \leq-m^{\prime} \zeta^{\varepsilon_{0}}(t) \mathscr{E}^{\varepsilon_{0}+1}(t)
$$

Integration over $\left(t_{0}, t\right)$ leads to for some constant $m^{\prime}>0$ such that

$$
\mathscr{L}_{1}(t) \leq m^{\prime}\left(1+\int_{t_{0}}^{t} \zeta^{\varepsilon_{0}+1}(p) d p\right)^{-1 / \varepsilon_{0}} .
$$

The equivalence of $\mathscr{L}_{1}(t)$ and $\mathscr{E}$ completes Proof of Theorem 8 .

Remark 12.

(1) We mention here that we have coupled our system without the classical way, i.e., our idea is not to couple equations in the logarithmic nonlinear terms

(2) Most contribution here is to obtain our nonexistence result with less conditions on the viscoelastic terms

\section{Data Availability}

No data were used in this study.

\section{Conflicts of Interest}

The authors declare that they have no competing interests.

\section{Authors' Contributions}

The authors contributed equally and significantly in writing this paper. All authors read and approved the final manuscript.

\section{References}

[1] X. Han, "Global existence of weak solutions for a logarithmic wave equation arising from Q-ball dynamics," Bulletin of the Korean Mathematical Society, vol. 50, no. 1, pp. 275-283, 2013.

[2] W. Lian and R. Xu, "Global well-posedness of nonlinear wave equation with weak and strong damping terms and logarithmic source term," Advances in Nonlinear Analysis, vol. 9, no. 1, pp. 613-632, 2020.

[3] M. Al-Gharabli, "New general decay results for a viscoelastic plate equation with a logarithmic nonlinearity," Boundary Value Problems, vol. 2019, no. 1, pp. 1-21, 2019.

[4] P. G. Papadopoulos and N. M. Stavrakakis, "Global existence and blow-up results for an equation of Kirchhoff type on $\mathbb{R}^{N}$," Topological Methods in Nonlinear Analysis, vol. 17, pp. 91109, 2001.

[5] T. Miyasita and K. Zennir, "A sharper decay rate for a viscoelastic wave equation with power nonlinearity," Mathematical Methods in the Applied Sciences, vol. 43, pp. 1138-1144, 2020.

[6] N. I. Karachalios and N. M. Stavrakakis, "Global existence and blow-up results for some nonlinear wave equations on $\mathbb{R}^{N}$," Advances in Difference Equations, vol. 6, 174 pages, 2001.

[7] L. Gross, "Logarithmic Sobolev inequalities," American Journal of Mathematics, vol. 97, no. 4, pp. 1061-1083, 1975.

[8] T. Cazenave and A. Haraux, "Équations d'évolution avec non linéarité logarithmique," Annales de la faculté des sciences de Toulouse Mathématiques, vol. 2, no. 1, pp. 21-51, 1980. 